\title{
Frontiers in phenotypic plasticity research: new questions about mechanisms, induced responses and ecological impacts
}

\author{
Jacintha Ellers $\cdot$ Josef F. Stuefer
}

Published online: 18 March 2010

(C) Springer Science+Business Media B.V. 2010

Phenotypic plasticity of organisms in response to environmental variability is a welldeveloped concept in ecology and evolutionary biology. It is currently seen as one of the prime mechanisms with which organisms can respond adaptively to environmental change. Despite its importance for evolutionary processes and the long standing history of empirical and theoretical research on plasticity, we are still far from fully understanding the causes and consequences of phenotypic plasticity. This issue of Evolutionary Ecology contains seven papers that employ the concept of phenotypic plasticity to tackle new questions at their respective fields of research. While introducing new concepts in an existing field may improve understanding of relevant phenomena, the reverse is equally valuable: studying phenotypic plasticity in new contexts may improve our understanding of mechanisms and evolution of phenotypic plasticity. To highlight these new frontiers in phenotypic plasticity research we briefly emphasize the importance of each paper, and its contributions to general plasticity theory.

One of the facets of phenotypic plasticity that have remained resistant to research is the underlying mechanisms allowing for the production of different phenotypes from a single genotype (Schlichting and Smith 2002). A critical step in the relationship between genotype and phenotype is transcription and translation. Environmental signals are important modulators of the transcriptional activity of genes, and altered gene expression has been linked to environmentally-induced phenotypes (Kent et al. 2009; Sumner et al. 2006). The presence of specific DNA sequences upstream of the coding region of a gene encodes binding sites for transcription factors that can promote or inhibit gene expression. Despite the ubiquity of this view, we do not know what reaction norms for gene expression look like and what the selective pressures shaping those reaction norms really are. Roelofs et al. (2010) review a possible mechanism responsible for transcriptional plasticity in stress responses within single or multiple generations. Gene promoters containing a core

\footnotetext{
J. Ellers $(\bowtie)$

Department of Animal Ecology, Institute of Ecological Science, Faculty of Earth and Life Sciences, VU University Amsterdam, De Boelelaan 1085, 1081 HV Amsterdam, The Netherlands e-mail: jacintha.ellers@falw.vu.nl

J. F. Stuefer

Netherlands Organization for Scientific Research, The Hague, The Netherlands
} 
promoter structure such as a TATA box are more capable of rapid and variable responses than TATA-less genes, providing the organism with a larger capacity to buffer environmental stress, thereby improving survival. Including the molecular level when considering environmentally-induced phenotypes enforces the idea that canalization of fitness traits such as survival is only achieved through plasticity in the underlying mechanisms (Richards et al. 2006; Liefting et al. 2009).

Environmental conditions can also induce phenotypic effects that last over multiple generations. Epigenetic processes such as DNA methylation control gene expression through modification of DNA by the addition of methyl groups to cytosine residues (Bender 2004). The discovery of functional DNA methylation systems in an increasing number of species suggests that the importance of epigenetic effects may have been severely underestimated in the past. Altering DNA methylation has been shown to modify many traits including caste determination in honeybees (Elango et al. 2009), coat colour in mice (Morgan et al. 1999), and the timing of flowering and floral symmetry in a variety of plant species (Burn et al. 1993; Cubas et al. 1999). In this issue, Bossdorf et al. (2010) extend the known effects of methylation to include effects on phenotypic plasticity. The application of the demethylating agent 5-azacytidine (5-azaC) to Arabidopsis genotypes revealed significant effects on the sensitivity of flowering time, size at flowering, and plant biomass to nutrient levels. For example, demethylated plants that grew at high nutrient levels flowered later than demethylated plants at low nutrient levels, while in non-demethylated plants there was a tendency for nutrients to have the opposite effect on flowering time. To understand plastic responses it may therefore be important to combine knowledge on genotype and epigenotype, but many of the details remain to be elucidated.

During life, an individual's phenotypic response to environmental cues need not be fixed. Often, the effect of environmental conditions is moderated by previous experience with such conditions, for example in the case of heat and cold hardening. Exposure to mild heat or cold stress reduces the lethality of subsequent, more severe temperature shocks (Feder and Hofmann 1999; Hoffmann et al. 2003; Bahrndorff et al. 2009). Similar conditioning can be observed for behavioural responses which can be modified through learning (Smid et al. 2007) or for immune responses, which are induced by previous contacts with a pathogen (Schmid-Hempel 2005). Two reviews highlight this aspect of plasticity. First, Heil (2010) reviews induced defence responses in plants in response to herbivory and plant pathogens. Although plastic responses are generally evolutionary favoured because of their reduced energetic costs compared to constitutive defence, not every attack on the plant is a reliable indicator of future damage by herbivores. In addition to a full-blown induced resistance response, Heil distinguishes priming of plants: primed tissues show no enhanced levels in their phenotypic resistance but once attacked, they are capable of expressing resistance traits more quickly and more strongly. Infestation or infection may therefore not lead to uniform induced responses, but depend on the (recent) history of biotic interactions of individual plants.

In a second contribution on this topic, Mery and Burns (2010) consider conditioning of behavioural responses. Organisms can change their behaviour in response to environmental change through a predetermined innate response or through learning, which modifies the response to environmental cues as a result of previous experience. Which of these options is evolutionary favoured depends on the predictability and reliability of environmental cues, but also on the costs associated with either strategy. Learning has been one of the few forms of plasticity for which clear costs have been found. Mery and Kawecki (2002) showed that fruit flies selected for improved learning ability had reduced larval competitive ability even when their learning and memory abilities were not 
challenged. Until now, plasticity theory does not usually account for the fact that reaction norms change with the conditions individuals are acclimated to (Terblanche and Chown 2006), whereas this phenomenon has been much better described in physiology and immunology.

In addition to knowledge on how evolution shapes optimal levels of plasticity, a pressing question concerns the integrative effects of plasticity in multiple traits. We need to develop analytical tools that can help predict the effects of phenotypic plasticity on processes and phenomena at larger scales. Hierarchical population models may provide a way to model trait-trait interactions and determine which plastic traits are vital for population dynamics (Jongejans et al. 2010). In a case study on plant responses to nutrient enrichment, Jongejans et al. clearly illustrate the potential of this approach. In four grassland herb species, they identified those traits whose plastic variation had the biggest effects on vital rates. Increased plant size and increased seed production at higher nutrient levels were the plastic responses that were most important at the population level. Interestingly, one of the four species showed no plasticity in these traits, and this species did relatively poor under changing nutrient conditions. This may have significant implications for the vulnerability of species to patterns of global environmental change.

Perhaps our biggest challenge now is to understand the ecological impact of plasticity (Agrawal 2001; Miner et al. 2005). Chown et al. (2010) extend this approach by considering plastic population responses at an even larger scale. They review methods to understand the ecological implications of physiological plasticity across large spatial and temporal scales within a landscape matrix. Will phenotypic plasticity promote or retard changes in geographic ranges associated with changing local conditions? Such approaches need to recognize that the higher levels of biological hierarchy are emergent from the population level, but also feed back to it.

Many studies on phenotypic plasticity have concentrated on individual species. This ignores the fact that species are part of complex interaction networks, in which species interactions may be condition-dependent (Relyea and Yurewicz 2002). Differential plasticity among species or trophic groups in response to environmental change may lead to phenological or spatial mismatch (Berg et al. 2010). Therefore we need to integrate the concept of plasticity into multitrophic relationships such as food webs or ecological communities. However, this can be a daunting task given the multitude of potential species interactions within communities, as well as the numerous individual traits that can show phenotypic plasticity. It would be most beneficial if we could modify existing models to include the effects of phenotypic plasticity. In this issue, Berg and Ellers (2010) make a notable attempt by including trait plasticity in a niche competition model to predict changes in species exclusion and coexistence. They arrive at specific predictions on the type of traits for which plasticity affects species interactions. For example, plasticity in resource uptake is predicted to affect the strength of competition between species and hence the potential for species exclusion. Although such models allow testable predictions, major advances are hampered by the lack of empirical data to verify these predictions.

The special issue underlines that although the concept of phenotypic plasticity is wellestablished, many new aspects remain to be discovered. It is mostly through an interdisciplinary approach that we can now advance our understanding of the more complex questions about phenotypic plasticity. We need to look beyond the traditional views on plasticity to answer those new questions, and show flexibility in adopting insights from other disciplines. 


\section{References}

Agrawal AA (2001) Phenotypic plasticity in the interactions and evolution of species. Science 294:321-326

Bahrndorff S, Marien J, Loeschcke V, Ellers J (2009) Dynamics of heat-induced thermal stress resistance and Hsp70 expression in the springtail, Orchesella cincta. Funct Ecol 23:233-239

Bender J (2004) DNA methylation and epigenetics. Ann Rev Plant Biol 55:41-68

Berg MP, Ellers J (2010) Trait plasticity in species interactions: a driving force of community dynamics. Evol Ecol 24 (in this issue). doi:10.1007/s10682-009-9347-8

Berg MP, Kiers ET, Driessen G et al (2010) Adapt or disperse: understanding species persistence in a changing world. Glob Change Biol 16:587-598

Bossdorf O, Arcuri D, Richards CL, Pigliucci M (2010) Experimental alteration of DNA methylation affects the phenotypic plasticity of ecologically relevant traits in Arabidopsis thaliana. Evol Ecol 24 (in this issue). doi:10.1007/s10682-010-9372-7

Burn JE, Bagnall DJ, Metzger JD, Dennis ES, Peacock WJ (1993) DNA methylation, vernalization, and the initiation of flowering. Proc Nat Acad Sci 90:287-291

Chown SL, Gaston KJ, van Kleunen M, Clusella-Trullas S (2010) Population responses within a landscape matrix: a macrophysiological approach to understanding climate change impacts. Evol Ecol 24 (in this issue). doi:10.1007/s10682-009-9329-x

Cubas P, Vincent C, Coen E (1999) An epigenetic mutation responsible for natural variation in floral symmetry. Nature 401:157-161

Elango N, Hunt BG, Goodisman MAD, Yi SV (2009) DNA methylation is widespread and associated with differential gene expression in castes of the honeybee, Apis mellifera. Proc Nat Acad Sci 106:11206-11211

Feder ME, Hofmann GE (1999) Heat-shock proteins, molecular chaperones, and the stress response: evolutionary and ecological physiology. Ann Rev Physiol 61:243-282

Heil M (2010) Plastic defence expression in plants. Evol Ecol 24 (in this issue). doi:10.1007/ s10682-009-9348-7

Hoffmann AA, Sorensen JG, Loeschcke V (2003) Adaptation of Drosophila to temperature extremes: bringing together quantitative and molecular approaches. J Thermal Biol 28:175-216

Jongejans E, Huber H, de Kroon H (2010) Scaling up phenotypic plasticity with hierarchical population models. Evol Ecol 24 (in this issue). doi:10.1007/s10682-009-9340-2

Kent CF, Daskalchuk T, Cook L, Sokolowski MB, Greenspan RJ (2009) The Drosophila foraging gene mediates adult plasticity and gene-environment interactions in behaviour, metabolites, and gene expression in response to food deprivation. Plos Genetics 5

Liefting M, Hoffmann AA, Ellers J (2009) Plasticity versus environmental canalization: population differences in thermal responses along a latitudinal gradient in Drosophila serrata. Evolution 63:1954-1963

Mery F, Burns JG (2010) Behavioural plasticity: an interaction between evolution and experience. Evol Ecol 24 (in this issue). doi:10.1007/s10682-009-9336-y

Mery F, Kawecki TJ (2002) Experimental evolution of learning ability in fruit flies. Proc Nat Acad Sci 99:14274-14279

Miner BG, Sultan SE, Morgan SG et al (2005) Ecological consequences of phenotypic plasticity. Trends Ecol Evol 20:685-692

Morgan HD, Sutherland HGE, Martin DIK, Whitelaw E (1999) Epigenetic inheritance at the agouti locus in the mouse. Nat Genet 23:314-318

Relyea RA, Yurewicz KL (2002) Predicting community outcomes from pair-wise interactions: integrating density- and trait-mediated effects. Oecologia 131:569-579

Richards CL, Bossdorf O, Muth NZ et al (2006) Jack of all trades, master of some? On the role of phenotypic plasticity in plant invasions. Ecol Lett 9:981-993

Roelofs D, Morgan J, Stürzenbaum S (2010) The significance of genome-wide transcriptional regulation in the evolution of stress tolerance. Evol Ecol 24 (in this issue). doi:10.1007/s10682-009-9345-x

Schlichting CD, Smith H (2002) Phenotypic plasticity: linking molecular mechanisms with evolutionary outcomes. Evol Ecol 16:189-211

Schmid-Hempel P (2005) Evolutionary ecology of insect immune defenses. Ann Rev Entomol 50:529-551

Smid HM, Wang GH, Bukovinszky T, Steidle JLM, Bleeker MAK, van Loon JJA, Vet LEM (2007) Speciesspecific acquisition and consolidation of long-term memory in parasitic wasps. Proc R Soc B 274:1539-1546

Sumner S, Pereboom JJM, Jordan WC (2006) Differential gene expression and phenotypic plasticity in behavioural castes of the primitively eusocial wasp, Polistes canadensis. Proc R Soc B 273:19-26

Terblanche JS, Chown SL (2006) The relative contributions of developmental plasticity and adult acclimation to physiological variation in the tsetse fly, Glossina pallidipes (Diptera, Glossinidae). J Exp Biol 209:1064-1073 